\title{
Gradient-Enhanced TROSY Described with Cartesian Product Operators
}

\author{
ERIK R.P. ZUIDERWEG, ${ }^{1}$ AIKATERINI ROUSAKI ${ }^{2}$ \\ ${ }^{1}$ Department of Biological Chemistry, University of Michigan, 1150 W. Medical Center Drive, Ann Arbor, MI 48109 \\ ${ }^{2}$ LSA Biophysics, University of Michigan, 930 N University Avenue, Ann Arbor, MI 48109
}

\begin{abstract}
TROSY, Transverse Relaxation Optimized Spectroscopy, was developed more than a decade ago. Since that time, the ${ }^{15} \mathrm{~N}-{ }^{1} \mathrm{H}$ HSOC-TROSY experiment has become the standard "fingerprint" correlation spectrum for proteins of high molecular weight. In addition, its implementation in protein triple resonance experiments has pushed the boundaries of NMR assignment up to about $100 \mathrm{kDa}$, making NMR a highly relevant technique in structural biology. TROSY exploits the dipole-CSA cross-correlated relaxation properties of the NH system and selects for the narrowest of the HSOC J-correlation quartet in both dimensions. The original publications and reviews of TROSY use shift operators and/or single transition product operators to describe the TROSY coherence pathways selections. In this review, we offer a familiar Cartesian product operator approach to comprehensively describe all of the events in the modern TROSY pulse sequence such as multiplet selection, gradient coherence selection, gradient quadrature, and sensitivity enhancement. (c) 2011 Wiley Periodicals, Inc. Concepts Magn Reson Part A 38: 280-288, 2011.
\end{abstract}

KEY WORDS: protein; NMR; cross-correlated relaxation

\section{INTRODUCTION}

TROSY (Transverse relaxation-optimized spectroscopy) is a technique that increases the resolution and

Received 18 July 2011; revised 13 September 2011; accepted 27 September 2011

Present address of Aikaterini Rousaki: Institute of Organic Chemistry and Chemical Biology, Goethe University, Max-von-Laue-Str. 7, Frankfurt, DE 60438.

Correspondence to: Erik R. P. Zuiderweg, E-mail: zuiderwe@ umich.edu

Concepts in Magnetic Resonance Part A, Vol. 38A(6) 280-288 (2011) Published online in Wiley Online Library (wileyonlinelibrary.com). DOI 10.1002/cmr.a.20228

(c) 2011 Wiley Periodicals, Inc. sensitivity of heteronuclear NMR experiments on especially larger molecular systems at higher magnetic field strengths. The technique has been applied to two-dimensional ${ }^{1} \mathrm{H}^{-15} \mathrm{~N}$ chemical shift correlation in proteins (1) (optimal at 800-900 MHz) and ${ }^{1} \mathrm{H}_{-}{ }^{13} \mathrm{C}$ chemical shift correlation of aromatic residues $(2,3)$ (optimal at 500-700 MHz). The TROSY effect is also exploited to make sensitivity gains in triple resonance protein assignment experiments (4). These experiments have all in common that the coherence resides for a long time on the ${ }^{15} \mathrm{~N}$ transition. The selection for the narrow, and, therefore, long-lived TROSY component of that transition greatly enhances sensitivity of these experiments, even for small proteins at moderate fields. One may say that the biggest impact of TROSY was to extend the feasibility of protein triple-resonance assignment from 20 to $100 \mathrm{kDa}$. 


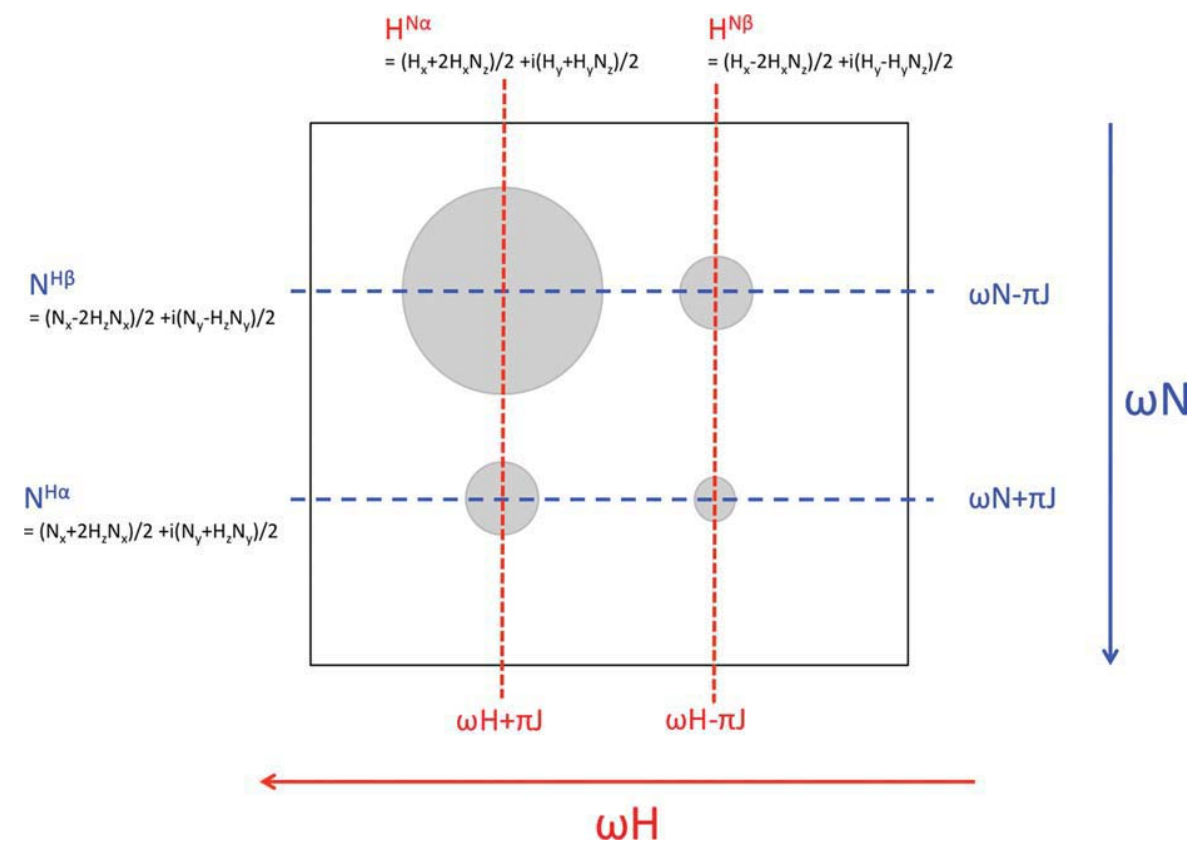

Figure 1 Definitions of the transitions in the ${ }^{1} \mathrm{H}_{-}{ }^{15} \mathrm{~N}$ quartet. The $\omega \mathrm{H}$ frequency becomes larger and more negative to the left $(\gamma \mathrm{H}$ is negative), while the $\omega \mathrm{N}$ becomes larger and more positive to the bottom $\left(\gamma \mathrm{N}\right.$ is positive). The ${ }^{1} J_{\mathrm{HN}}$ scalar coupling is positive $(12,19)$.

In addition to the original publications, several comprehensive reviews of TROSY are available. However, none of these papers use the familiar Cartesian product operators $(5,6)$ to describe the TROSY coherence pathway selections. In addition, the modern forms of the TROSY experiment accomplish a remarkably efficient coherence path selection using pulsed-field gradients. The pulse sequence selects the TROSY component and suppresses the anti-TROSY component in both dimensions, achieves sensitivity enhancement (preservation of coherence pathways according to Rance (7) and Kay (8) accomplishes quadrature detection in the ${ }^{15} \mathrm{~N}$ dimension, and suppresses axial peaks. All this is achieved with a sequence that theoretically needs only one transient per $t_{1}$ value and quadrature component and which preserves the solvent resonance Z-magnetization. In this paper we describe all of these events using the familiar Cartesian product operators.

\section{Cross-Correlated Relaxation in HSOC}

Crosspeaks in heteronuclear J-correlation experiments such as HSQC are doublets in both dimensions. Only by decoupling in both dimensions does the familiar singlet cross peak at the central chemical shift positions arise. Without this decoupling, one also observes another effect: the linewidths of the four components of the quartet are all different from each other, as is shown in Fig. 1.

The differential broadening in the ${ }^{15} \mathrm{~N}$ doublet is caused by constructive and destructive interference between the ${ }^{1} \mathrm{H}_{-}{ }^{15} \mathrm{~N}$ dipole-dipole and the ${ }^{15} \mathrm{~N}$ chemical shift anisotropy (CSA) relaxation mechanisms and in the ${ }^{1} \mathrm{H}$ doublet by interference between the ${ }^{1} \mathrm{H}-{ }^{15} \mathrm{~N}$ dipole-dipole relaxation and the ${ }^{1} \mathrm{H}$ CSA relaxation mechanisms. The interference is also known as CSA-dipolar cross-correlated relaxation.

A pictorial explanation of the mechanism of dipolar-dipolar/CSA relaxation interference which gives rise to the different relaxation rates is presented in Fig. $2(9-11)$.

The ${ }^{15} \mathrm{~N}$ nucleus is subjected to a local field composed of the following two components. The first is the external magnetic field, attenuated by chemical shielding, and the second is the local magnetic field due to the neighboring proton. Both local fields are dependent on the orientation of the molecular fragment in the field; the dipolar interaction because the magnetic dipoles remain locked to the external field no matter what the orientation is, and the shielding, because it is anisotropic, or different, for different orientations because the molecular orbitals harboring the electrons that cause the shielding are anisotropic. 


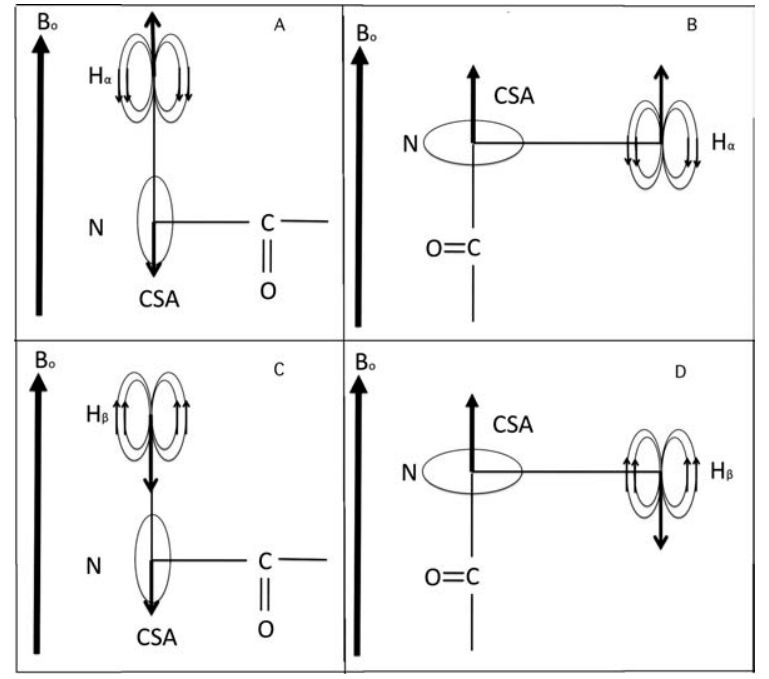

Figure 2 Origin of dipolar-CSA cross-correlated relaxation: correlated fluctuations of the CSA and dipolar induced fields.

In Figs. 2(A,C), the ${ }^{15} \mathrm{~N}$ nucleus is shielded more-than-average (local field arrow down), while in Figs. 2(B,D) it is shielded less-than-average (local field arrow up). In both Figs. 2(A,B), the local field at the ${ }^{15} \mathrm{~N}$ nucleus caused by the dipolar field of the ${ }^{1} \mathrm{H}$ nucleus in the alpha state, counters the CSA local field. That is, if the molecule rotates, the variation in CSA shielding is compensated by the variation in dipolar field. In the ideal case, no net variation occurs, so that both the CSA and dipolar relaxation are canceled. This gives rise to the narrow line in the ${ }^{15} \mathrm{~N}-{ }^{1} \mathrm{H}$ scalar coupling doublet.

In both Figs. 2(C,D), the local field at the ${ }^{15} \mathrm{~N}$ nucleus caused by the dipolar field of the ${ }^{1} \mathrm{H}$ nucleus in the beta state, reinforces the CSA local field. Hence, if the molecule rotates, the variation in CSA shielding is reinforced by the variation in dipolar field. This gives rise to efficient $R_{2}$ relaxation and to the broad line in the ${ }^{15} \mathrm{~N}-{ }^{1} \mathrm{H}$ scalar coupling doublet.

In a completely analogous fashion, dipolar-dipolar/CSA relaxation interference also occurs for the ${ }^{1} \mathrm{H}$ nucleus, subject to ${ }^{1} \mathrm{H} \mathrm{CSA}$ and the dipolar field generated by the ${ }^{15} \mathrm{~N}$ nucleus. The above description is (approximately) true only for the large-molecule limit. For small molecules, additional high-frequency relaxation effects spoil the TROSY effect. Quantitatively, the large-molecule $R_{2}$ relaxation rates for the four frequency components of the quartet of Fig. 1 are given in Table 1.
The symbols in Table 1 are:

$$
\begin{gathered}
\mathrm{dd}=\frac{1}{8}\left(\frac{\mu_{0}}{4 \pi}\right)^{2}\left(\frac{\gamma_{H} \gamma_{N} \hbar}{r_{H N}^{3}}\right)^{2} 4 J(0) \quad \text { (positive) } \\
\operatorname{csa}_{\mathrm{N}}=\frac{1}{18}\left(\omega_{N} \Delta \sigma_{N}\right)^{2} 4 J(0) \quad \text { (positive) } \\
\operatorname{csa}_{\mathrm{H}}=\frac{1}{18}\left(\omega_{H} \Delta \sigma_{H}\right)^{2} 4 J(0) \quad \text { (positive) } \\
\mathrm{dc}_{\mathrm{H}}=\frac{1}{6}\left(\frac{\mu_{0}}{4 \pi}\right) \frac{\hbar \gamma_{H} B_{0} \Delta \sigma_{H} \gamma_{H} \gamma_{N}}{r_{H N}^{3}}\{4 J(0)\} \quad \text { (positive) } \\
\mathrm{dc}_{\mathrm{N}}=\frac{1}{6}\left(\frac{\mu_{0}}{4 \pi}\right) \frac{\hbar \gamma_{N} B_{0} \Delta \sigma_{N} \gamma_{H} \gamma_{N}}{r_{H N}^{3}}\{4 J(0)\} \quad \text { (negative) }
\end{gathered}
$$

The expressions for the relaxation rates were derived in (11). It is assumed that the protein is large such that $J(0) \gg J(\omega)$ and that the axial chemical shift tensors are aligned along the $\mathrm{NH}$ bond vector. $J(0)$ is the spectral density at $\omega=0$ and is defined here as: $J(\omega)=(2 / 5)\left[\tau_{\mathrm{c}} /\left(1+\omega^{2} \tau_{\mathrm{c}}^{2}\right)\right]=(2 / 5) \tau_{\mathrm{c}} . B_{\mathrm{o}}$ is the static magnetic field, and $\Delta \sigma_{\mathrm{H}, \mathrm{N}}$ is the chemical shift anisotropy for either the proton or the nitrogen, $\gamma_{\mathrm{H}}$ is the ${ }^{1} \mathrm{H}$ gyromagnetic ratio [negative (12)], $\gamma_{\mathrm{N}}$ is the ${ }^{15} \mathrm{~N}$ gyromagnetic ratio [positive (12)]. $\frac{\mu_{0}}{4 \pi}=10^{-7}$, dimensionless, is the magnetic permittivity of vacuum, inserted to allow use of SI units for the other parameters.

The equations show that the TROSY effect is dependent on magnetic field (as a result of the magnetic-field dependency of the CSA relaxation). Figures 3(A,B) show calculations for the protein amide ${ }^{15} \mathrm{~N}$ TROSY and ${ }^{1} \mathrm{H}$ TROSY effects, using the best literature values for rhombic CSA tensors (13) and including the fast frequency relaxation terms. The maximum effect is obtained when $d d+c s a_{\mathrm{H}}=$ $\left|d c_{\mathrm{H}}\right|$ or $d d+c s a_{\mathrm{N}}=\left|d c_{\mathrm{N}}\right|$. Figure 3 shows that this occurs approximately simultaneously for the protein ${ }^{1} \mathrm{H}^{15} \mathrm{~N}$ system at $B_{0}=18-20 \mathrm{~T}\left(\omega_{\mathrm{H}}=800-1000\right.$ $\mathrm{MHz}$ ). This is a fortuitous consequence of the fact that $\gamma_{H} \cong-10 \times \gamma_{N}$ and that $\Delta \sigma_{H} \cong 0.1 \times \Delta \sigma_{N}$ for the amide spin system. The figures show that the

Table 1 Cross-Correlated Relaxation Rates from (1) and (11)

\begin{tabular}{lll}
\hline Transition Frequencies & \multicolumn{1}{c}{$\begin{array}{l}\text { Approximate R2 } \\
\text { Relaxation Rate }\end{array}$} \\
\hline$H_{\mathrm{N} \beta}$ & $\omega_{\mathrm{H}}-\pi J$ & $4 J(0)\left[\mathrm{dd}+\mathrm{csa}_{\mathrm{H}}-\mathrm{dc}_{\mathrm{H}}\right]{ }^{1} \mathrm{H}$ TROSY \\
$H_{\mathrm{N} \alpha}$ & $\omega_{\mathrm{H}}+\pi J$ & $4 J(0)\left[\mathrm{dd}+\mathrm{csa}_{\mathrm{H}}+\mathrm{dc}_{\mathrm{H}}\right]{ }^{1} \mathrm{H}$ anti-TROSY \\
$N_{\mathrm{H} \alpha}$ & $\omega_{\mathrm{N}}+\pi J$ & $4 J(0)\left[\mathrm{dd}+\mathrm{csa}_{\mathrm{N}}+\mathrm{dc}_{\mathrm{N}}\right]{ }^{15} \mathrm{~N}$ TROSY \\
$N_{\mathrm{H} \beta}$ & $\omega_{\mathrm{N}}-\pi J$ & $4 J(0)\left[\mathrm{dd}+\mathrm{csa}_{\mathrm{N}}-\mathrm{dc}_{\mathrm{N}}\right]{ }^{15} \mathrm{~N}$ anti-TROSY \\
\hline
\end{tabular}



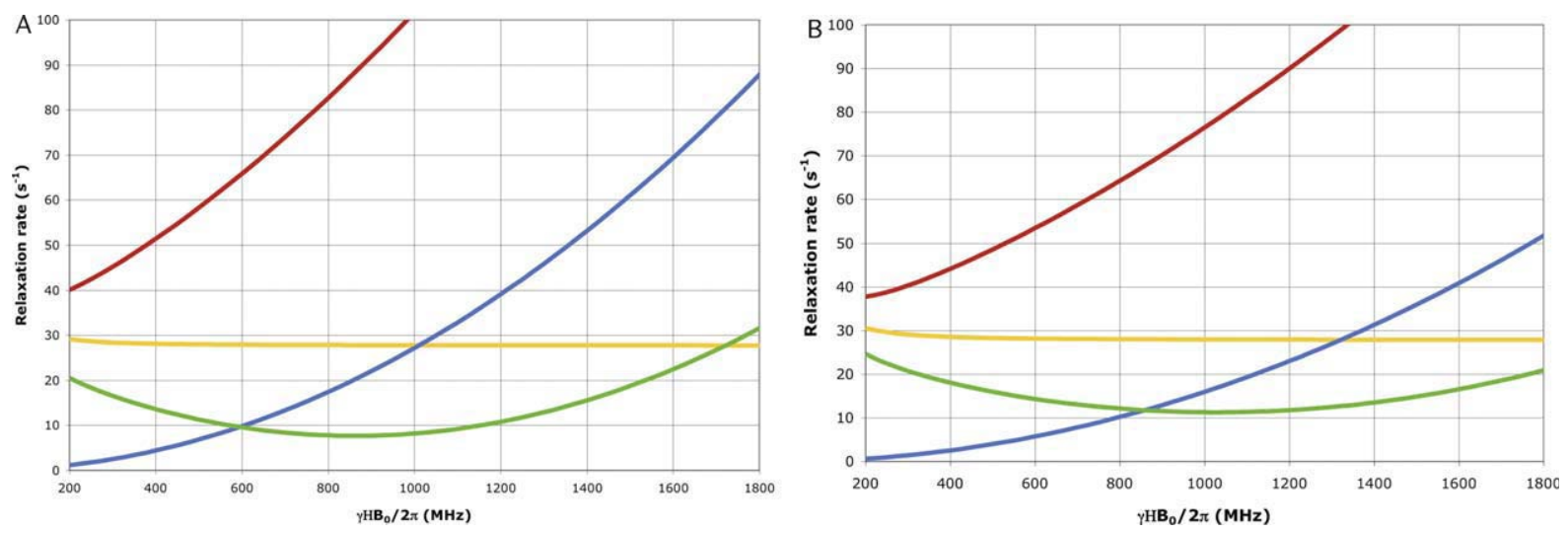

Figure 3 (A) The ${ }^{15} \mathrm{~N}$ TROSY effect as a function of spectrometer ${ }^{1} \mathrm{H}$ frequency calculated for a rotational correlation time of $30 \mathrm{~ns}$. Yellow, the ${ }^{15} \mathrm{~N}-{ }^{1} \mathrm{H}$ dipolar relaxation; blue, the ${ }^{15} \mathrm{~N}$ CSA relaxation; green, TROSY; red, anti-TROSY. (B) The ${ }^{1} \mathrm{H}$ TROSY effect as a function of spectrometer ${ }^{1} \mathrm{H}$ frequency. Yellow, the ${ }^{15} \mathrm{~N}-{ }^{1} \mathrm{H}$ dipolar relaxation; blue, the ${ }^{1} \mathrm{H}$ CSA relaxation; green, TROSY; red, anti-TROSY.

interference effects are already considerable at lower magnetic fields. Other calculations show that aromatic ${ }^{13} \mathrm{C}^{1} \mathrm{H}$ systems have a maximum TROSY benefit at $\sim 600 \mathrm{MHz}$, while guanine ${ }^{15} \mathrm{~N}^{1} \mathrm{H}$ TROSY is optimal at $\sim 1200 \mathrm{MHz}$ and thymine ${ }^{15} \mathrm{~N}^{1} \mathrm{H}$ TROSY is very flat but optimal at $\sim 1500 \mathrm{MHz}$.

\section{The TROSY Experiment}

While the existence of relaxation interference was known for decades (14), scientists ${ }^{1}$ at the ETH at Zurich, Switzerland, recognized its potential in the 1990s when large NMR magnets $(700 \mathrm{MHz}$ and beyond) became available. The usual decoupling techniques in HSQC mix the narrow and broad components. Especially for large proteins the broad components of the quartet are so broad that decoupling degrades the spectrum to such extent that it cannot be used anymore. Next it was realized that the narrow line of a ${ }^{15} \mathrm{~N}-{ }^{1} \mathrm{H}$ HSQC of proteins could become very narrow at $800-900 \mathrm{MHz}$, mostly independent of molecular weight. Two more steps were needed: the first, large advance was made by Pervushin (1), who developed a pulse sequence element that selects for the narrow component of the quartet. The next, smaller step, was to combine the pulse sequence element with gradient-sensitivity enhancement (15-18).

\section{Cartesian TROSY and Anti-TROSY Terms}

The TROSY the anti-TROSY terms that we will discuss in this paper are given in Fig. 1. The slowest relaxing rates give the (double) TROSY term and it is composed of the transitions $\mathrm{HN} \beta$ and $\mathrm{NH} \alpha$ for the hydrogen and nitrogen spins, respectively, conform the signs of the relaxation rates defined above. The basic idea behind the Cartesian description of the TROSY experiment is the addition and subtraction of the Cartesian $N_{\mathrm{x}}$ coherences from the Cartesian term $2 N_{\mathrm{x}} H_{\mathrm{z}}$ in order to create a Cartesian representation of the TROSY and the anti-TROSY terms. This is illustrated with vectors diagrams in Fig. 4. To show the rigorous validity of this transformation, we start by recalling the definition of the density operator (i.e., the state descriptor of populations and transitions) which underlies the Cartesian product operators (6)

$$
\left[\begin{array}{ccccc}
\text { state } & H_{\alpha} N_{\alpha} & H_{\alpha} N_{\beta} & H_{\beta} N_{\alpha} & H_{\beta} N_{\beta} \\
H_{\alpha} N_{\alpha} & \text { pop } & N^{H \alpha} & H^{N \alpha} & D Q \\
H_{\alpha} N_{\beta} & N^{H \alpha} & \text { pop } & Z Q & H^{N \beta} \\
H_{\beta} N_{\alpha} & H^{N \alpha} & Z Q & \text { pop } & N^{H \beta} \\
H_{\beta} N_{\beta} & D Q & H^{N \beta} & N^{H \beta} & \text { pop }
\end{array}\right]
$$

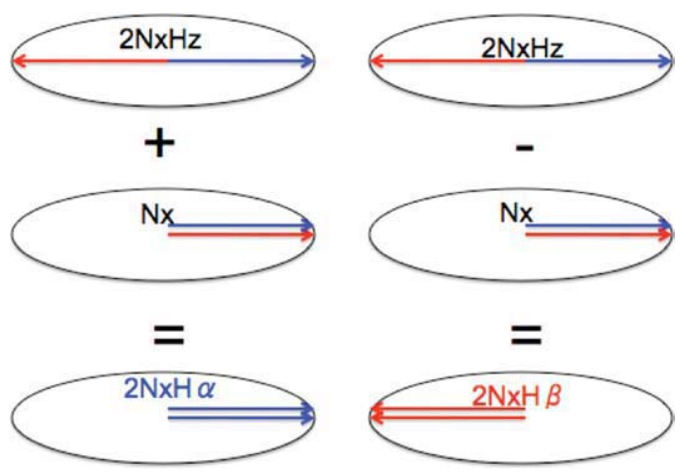

Figure 4 Decomposition of ${ }^{15} \mathrm{~N}$ coherence in TROSY and anti-TROSY components. 
The Cartesian product operators $H_{\mathrm{x}}, N_{\mathrm{x}}, H_{\mathrm{z}}$, and $N_{\mathrm{z}}$ are defined in these density matrices as

$$
\begin{aligned}
& H_{x} \equiv \frac{1}{2}\left[\begin{array}{llll}
0 & 0 & 1 & 0 \\
0 & 0 & 0 & 1 \\
1 & 0 & 0 & 0 \\
0 & 1 & 0 & 0
\end{array}\right] \\
& H_{z} \equiv \frac{1}{2}\left[\begin{array}{cccc}
1 & 0 & 0 & 0 \\
0 & 1 & 0 & 0 \\
0 & 0 & -1 & 0 \\
0 & 0 & 0 & -1
\end{array}\right] \\
& N_{x} \equiv \frac{1}{2}\left[\begin{array}{llll}
0 & 1 & 0 & 0 \\
1 & 0 & 0 & 0 \\
0 & 0 & 0 & 1 \\
0 & 0 & 1 & 0
\end{array}\right] N_{z} \equiv \frac{1}{2}\left[\begin{array}{cccc}
1 & 0 & 0 & 0 \\
0 & -1 & 0 & 0 \\
0 & 0 & 1 & 0 \\
0 & 0 & 0 & -1
\end{array}\right]
\end{aligned}
$$

The Cartesian "anti-phase" states are derived from these by tensor multiplication:

$$
\begin{aligned}
& 2 H_{x} N_{z}= \frac{1}{2}\left[\begin{array}{cccc}
0 & 0 & 1 & 0 \\
0 & 0 & 0 & -1 \\
1 & 0 & 0 & 0 \\
0 & -1 & 0 & 0
\end{array}\right] \\
& 2 H_{z} N_{x}=\frac{1}{2}\left[\begin{array}{cccc}
0 & 1 & 0 & 0 \\
1 & 0 & 0 & 0 \\
0 & 0 & 0 & -1 \\
0 & 0 & -1 & 0
\end{array}\right]
\end{aligned}
$$

Finally, the Cartesian TROSY and anti-TROSY transitions are obtained by addition and subtraction:

$$
\begin{aligned}
& \left(H_{x}+2 H_{x} N_{z}\right) / 2=\frac{1}{2}\left[\begin{array}{llll}
0 & 0 & 1 & 0 \\
0 & 0 & 0 & 0 \\
1 & 0 & 0 & 0 \\
0 & 0 & 0 & 0
\end{array}\right] \equiv H_{x}^{N \alpha} \\
& \left(H_{x}-2 H_{x} N_{z}\right) / 2=\frac{1}{2}\left[\begin{array}{llll}
0 & 0 & 0 & 0 \\
0 & 0 & 0 & 1 \\
0 & 0 & 0 & 0 \\
0 & 1 & 0 & 0
\end{array}\right] \equiv H_{x}^{N \beta} \\
& \left(N_{x}+2 H_{z} N_{x}\right) / 2=\frac{1}{2}\left[\begin{array}{llll}
0 & 1 & 0 & 0 \\
1 & 0 & 0 & 0 \\
0 & 0 & 0 & 0 \\
0 & 0 & 0 & 0
\end{array}\right] \equiv N_{x}^{H \alpha} \\
& \left(N_{x}-2 H_{z} N_{x}\right) / 2=\frac{1}{2}\left[\begin{array}{llll}
0 & 0 & 0 & 0 \\
0 & 0 & 0 & 0 \\
0 & 0 & 0 & 1 \\
0 & 0 & 1 & 0
\end{array}\right] \equiv N_{x}^{H \beta}
\end{aligned}
$$

Table 2 Product Operator Transformations Using Definitions from Sorensen (6)

\begin{tabular}{l}
\hline$I_{z} \stackrel{\text { pulse } \beta x}{\longrightarrow} I_{z} \cos (\beta)-I_{y} \sin (\beta)$ \\
$I_{z} \stackrel{\text { pulse } \beta y}{\longrightarrow} I_{z} \cos (\beta)+I_{x} \sin (\beta)$ \\
$I_{x} \stackrel{\text { pulse } \beta y}{\longrightarrow} I_{x} \cos (\beta)-I_{z} \sin (\beta)$ \\
$I_{x} \stackrel{\text { shift }}{\longrightarrow} I_{x} \cos (\Omega t)+I_{y} \sin (\Omega t)$ \\
$I_{x} \stackrel{\text { gradient }}{\longrightarrow} I_{x} \cos \left(\gamma B_{z} t\right)+I_{y} \sin \left(\gamma B_{z} t\right)$ \\
$I_{x} \stackrel{\text { scalar }}{\longrightarrow} I_{x} \cos (\pi J t)+2 I_{y} S_{z} \sin (\pi J t)$ \\
$2 I_{x} S_{z} \stackrel{\text { scalar }}{\longrightarrow} 2 I_{x} S_{z} \cos (\pi J t)+I_{y} \sin (\pi J t)$ \\
$I_{y} \stackrel{\text { pulse } \beta x}{\longrightarrow} I_{y} \cos (\beta)+I_{z} \sin (\beta)$ \\
$I_{y} \stackrel{\text { shift }}{\longrightarrow} I_{y} \cos (\Omega t)-I_{x} \sin (\Omega t)$ \\
$I_{y} \stackrel{\text { gradient }}{\longrightarrow} I_{y} \cos \left(\gamma B_{z} t\right)-I_{x} \sin \left(\gamma B_{z} t\right)$ \\
$I_{y} \stackrel{\text { scalar }}{\longrightarrow} I_{y} \cos (\pi J t)-2 I_{x} S_{z} \sin (\pi J t)$ \\
$2 I_{y} S_{z} \stackrel{\text { scalar }}{\longrightarrow} 2 I_{y} S_{z} \cos (\pi J t)-I_{x} \sin (\pi J t)$ \\
$2 I_{x} S_{x}, 2 I_{y} S_{x}, 2 I_{x} S_{y}, 2 I_{y} S_{y}, \operatorname{no}$ evolution under $J_{I S}$ \\
\hline
\end{tabular}

Note the effect of positive $\left({ }^{15} \mathrm{~N}\right)$ or negative $\left({ }^{1} \mathrm{H},{ }^{13} \mathrm{C}\right)$ gyromagnetic ratio on the transformations of the coherences.

Similarly,

$$
\begin{array}{ll}
\left(H_{y}+2 H_{y} N_{z}\right) / 2 \equiv H_{y}^{N \alpha} & \left(H_{y}-2 H_{y} N_{z}\right) / 2 \equiv H_{y}^{N \beta} \\
\left(N_{y}+2 H_{z} N_{y}\right) / 2 \equiv N_{y}^{H \alpha} & \left(N_{y}-2 H_{z} N_{y}\right) / 2 \equiv N_{y}^{H \beta}
\end{array}
$$

We can now use the Cartesian product operator rules (6) to compute the effects of pulses and delays on the TROSY and anti-TROSY coherences. For convenience, these rules are reproduced in Table 2 .

\section{Description of the Selection Element in the TROSY Experiment}

A basic pulse sequence of a sensitivity-enhanced TROSY experiment is shown in Fig. 5. Our analysis starts with the $2 N_{\mathrm{x}} H_{\mathrm{z}}$ anti-phase coherence after the first INEPT transfer, which can now be recognized as a sum of TROSY and anti-TROSY terms:

$$
2 N_{x} H_{z}=\left(2 N_{x} H_{z}+N_{x}+2 N_{x} H_{z}-N_{x}\right) / 2
$$

Terms developing from scalar coupling refocusing during $t_{1}$ can also be written as sum of TROSY and anti-TROSY terms:

$$
N_{x}=\left(2 N_{x} H_{z}+N_{x}-2 N_{x} H_{z}+N_{x}\right) / 2
$$




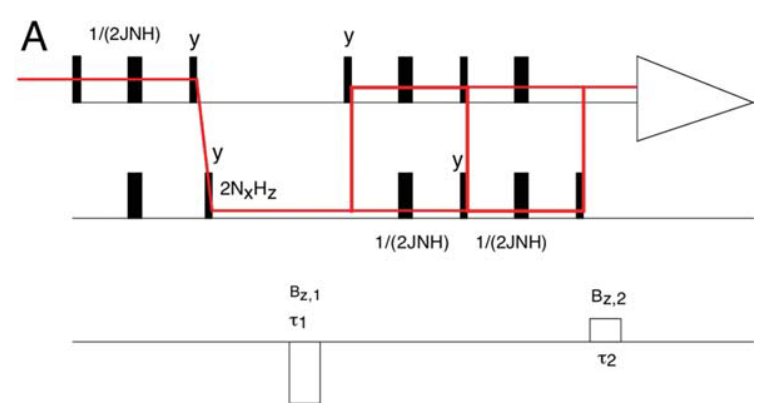

(h) The ${ }^{15} \mathrm{~N}$ y-phase anti-TROSY term in the anti echo-sequence

This is carried out here.

(a) The fate of the ${ }^{15} \mathrm{~N} x$-phase TROSY term in the echo-sequence

$$
\begin{aligned}
& 2 N_{x} H_{z}+N_{x} \stackrel{\text { Gradient@ location z }}{\longrightarrow}\left(2 N_{x} H_{z}+N_{x}\right) \\
& \times \cos \gamma_{N} B_{z, 1} \tau_{1}+\left(2 N_{y} H_{z}+N_{y}\right) \sin \gamma_{N} B_{z, 1} \tau_{1} \\
& \stackrel{900_{y}^{H}}{\longrightarrow}\left(2 N_{x} H_{x}+N_{x}\right) \cos \gamma_{N} B_{z, 1} \tau_{1} \\
& +\left(2 N_{y} H_{x}+N_{y}\right) \sin \gamma_{N} B_{z, 1} \tau_{1} \\
& \stackrel{J N H}{\longrightarrow}\left(2 N_{x} H_{x}+2 N_{y} H_{z}\right) \cos \gamma_{N} B_{z, 1} \tau_{1} \\
& +\left(2 N_{y} H_{x}-2 N_{x} H_{z}\right) \sin \gamma_{N} B_{z, 1} \tau_{1} \\
& \stackrel{90_{-x}^{H}}{\longrightarrow}\left(2 N_{x} H_{x}+2 N_{y} H_{y}\right) \cos \gamma_{N} B_{z, 1} \tau_{1} \\
& +\left(2 N_{y} H_{x}-2 N_{x} H_{y}\right) \sin \gamma_{N} B_{z, 1} \tau_{1} \\
& \stackrel{90_{y}^{N}}{\longrightarrow}\left(-2 N_{z} H_{x}+2 N_{y} H_{y}\right) \cos \gamma_{N} B_{z, 1} \tau_{1} \\
& +\left(2 N_{y} H_{x}+2 N_{z} H_{y}\right) \sin \gamma_{N} B_{z, 1} \tau_{1} \\
& \stackrel{J N H}{\longrightarrow}\left(-H_{y}+2 N_{y} H_{y}\right) \cos \gamma_{N} B_{z, 1} \tau_{1} \\
& +\left(2 N_{y} H_{x}-H_{x}\right) \sin \gamma_{N} B_{z, 1} \tau_{1} \\
& \stackrel{90_{x}^{N}}{\longrightarrow}\left(-H_{y}+2 N_{z} H_{y}\right) \cos \gamma_{N} B_{z, 1} \tau_{1} \\
& +\left(2 N_{z} H_{x}-H_{x}\right) \sin \gamma_{N} B_{z, 1} \tau_{1} \\
& \text { Gradient@locationz } \\
& \left(-H_{y}+2 N_{z} H_{y}\right) \cos \gamma_{N} B_{z, 1} \tau_{1} \cos \gamma_{H} B_{z, 2} \tau_{2} \\
& +\left(H_{x}-2 N_{z} H_{x}\right) \cos \gamma_{N} B_{z, 1} \tau_{1} \sin \gamma_{H} B_{z, 2} \tau_{2} \\
& +\left(2 N_{z} H_{x}-H_{x}\right) \sin \gamma_{N} B_{z, 1} \tau_{1} \cos \gamma_{H} B_{z, 2} \tau_{2} \\
& +\left(2 N_{z} H_{y}-H_{y}\right) \sin \gamma_{N} B_{z, 1} \tau_{1} \sin \gamma_{H} B_{z, 2} \tau_{2} \\
& \text { if } \gamma_{N} B_{z, 1} \tau_{1}=\gamma_{H} B_{z, 2} \tau_{2}=\varphi_{Z} \\
& \left(-H_{y}+2 N_{z} H_{y}\right)\left(\cos \varphi_{Z} \cos \varphi_{Z}+\sin \varphi_{Z} \sin \varphi_{Z}\right) \\
& =-\left(H_{y}-2 N_{z} H_{y}\right)
\end{aligned}
$$

We obtain that the slow-relaxing ${ }^{15} \mathrm{~N}\left(2 N_{x} H_{z}+N_{x}\right)$ TROSY term transfers into the slow-relaxing ${ }^{1} \mathrm{H}$ $-\left(H_{y}-2 H_{y} N_{z}\right)($ TROSY) term.

Note that the transfer is complete for every part of the sample, i.e., it is independent of the value of $\varphi_{z}$, which does vary over the sample.

When one uses $\tau_{1}=\tau_{2}$, the condition $\gamma_{N} B_{z, 1} \tau_{1}=$ $\gamma_{H} B_{z, 2} \tau_{2}$ requires that $B_{z, 1} \cong-10 \times B_{z, 2}$, since $\gamma_{N} \cong-0.1 \times \gamma_{H}$.

(b) In the ${ }^{15} \mathrm{~N}$ x-phase anti-TROSY term in the echo-sequence and subsequent derivations, we leave it to the reader to carry out the sign changes and show that one obtains during the decoding gradient: 


$$
\begin{aligned}
& 2 N_{x} H_{z}-N_{x} \stackrel{\text { gradient, delays, pulses, gradient }}{\longrightarrow} \\
& \left(-H_{y}-2 N_{z} H_{y}\right) \cos \gamma_{N} B_{z, 1} \tau_{1} \cos \gamma_{H} B_{z, 2} \tau_{2} \\
& +\left(H_{x}+2 N_{z} H_{x}\right) \cos \gamma_{N} B_{z, 1} \tau_{1} \sin \gamma_{H} B_{z, 2} \tau_{2} \\
& +\left(2 N_{z} H_{x}+H_{x}\right) \sin \gamma_{N} B_{z, 1} \tau_{1} \cos \gamma_{H} B_{z, 2} \tau_{2} \\
& +\left(2 N_{z} H_{y}+H_{y}\right) \sin \gamma_{N} B_{z, 1} \tau_{1} \sin \gamma_{H} B_{z, 2} \tau_{2} \\
& \text { if } \gamma_{N} B_{z, 1} \tau_{1}=\gamma_{H} B_{z, 2} \tau_{2}=\varphi_{Z} \\
& \begin{array}{l}
\left(2 N_{z} H_{y}+H_{y}\right)\left(\sin \varphi_{Z} \sin \varphi_{Z}-\cos \varphi_{Z} \cos \varphi_{Z}\right) \\
\quad+2\left(H_{x}+2 N_{z} H_{x}\right) \cos \varphi_{Z} \sin \varphi_{Z}
\end{array}
\end{aligned}
$$

The ${ }^{15} \mathrm{~N}$ anti-TROSY component transfers to the ${ }^{1} \mathrm{H}$ anti-TROSY component. However, the amount of transfer is dependent on the value of $\varphi_{z}$, which, because it is caused by a field gradient, varies over the sample.

One can easily show that

$$
\int_{0}^{2 n \pi} \cos \varphi_{Z} \sin \varphi_{Z} d \varphi_{Z}=0
$$

so that the ${ }^{15} \mathrm{~N}$ anti-TROSY term and all that develops from it is suppressed by the gradient over the full sample.

(Note, though, if $\gamma_{N} B_{z, 1} \tau_{1}=-\gamma_{H} B_{z, 2} \tau_{2}$, that the ${ }^{15} \mathrm{~N}$ anti-TROSY term is converted to the ${ }^{1} \mathrm{H}$ TROSY term.)

(c) The ${ }^{15} \mathrm{~N}$ y-phase TROSY term in the echosequence:

$$
\begin{aligned}
& 2 N_{y} H_{z}+N_{y} \stackrel{\text { gradient, pulses, delays, gradient }}{\longrightarrow} \\
& \left(2 N_{z} H_{x}-H_{x}\right) \cos \gamma_{N} B_{z, 1} \tau_{1} \cos \gamma_{H} B_{z, 2} \tau_{2} \\
& +\left(2 N_{z} H_{y}-H_{y}\right) \cos \gamma_{N} B_{z, 1} \tau_{1} \sin \gamma_{H} B_{z, 2} \tau_{2} \\
& -\left(-H_{y}+2 N_{z} H_{y}\right) \sin \gamma_{N} B_{z, 1} \tau_{1} \cos \gamma_{H} B_{z, 2} \tau_{2} \\
& -\left(H_{x}-2 N_{z} H_{x}\right) \sin \gamma_{N} B_{z, 1} \tau_{1} \sin \gamma_{H} B_{z, 2} \tau_{2} \\
& \text { if } \gamma_{N} B_{z, 1} \tau_{1}=\gamma_{H} B_{z, 2} \tau_{2}=\varphi_{Z} \\
& 2 N_{z} H_{x}-H_{x}
\end{aligned}
$$

The slow-relaxing ${ }^{15} \mathrm{~N}\left(2 N_{y} H_{z}+N_{y}\right)$ TROSY term transfers into the slow-relaxing ${ }^{1} \mathrm{H}\left(H_{x}-2 H_{x} N_{z}\right)$ (TROSY) term. Hence, both $x$ and $y$ coherence components are preserved in agreement with the Rance-Kay methodology $(7,8)$.

(d) The ${ }^{15} \mathrm{~N}$ y-phase anti-TROSY in the echosequence:

$$
\begin{aligned}
& 2 N_{y} H_{z}-N_{y} \stackrel{\text { gradient, pulses, delays, gradient }}{\longrightarrow} \\
& \left(2 N_{z} H_{x}+H_{x}\right) \cos \gamma_{N} B_{z, 1} \tau_{1} \cos \gamma_{H} B_{z, 2} \tau_{2} \\
& +\left(2 N_{z} H_{y}+H_{y}\right) \cos \gamma_{N} B_{z, 1} \tau_{1} \sin \gamma_{H} B_{z, 2} \tau_{2} \\
& -\left(-H_{y}-2 N_{z} H_{y}\right) \sin \gamma_{N} B_{z, 1} \tau_{1} \cos \gamma_{H} B_{z, 2} \tau_{2} \\
& -\left(H_{x}+2 N_{z} H_{x}\right) \sin \gamma_{N} B_{z, 1} \tau_{1} \sin \gamma_{H} B_{z, 2} \tau_{2} \\
& \text { if } \gamma_{N} B_{z, 1} \tau_{1}=\gamma_{H} B_{z, 2} \tau_{2}=\varphi_{Z} \\
& \left(2 N_{z} H_{x}+H_{x}\right)\left(\cos \varphi_{Z} \cos \varphi_{Z}-\sin \varphi_{Z} \sin \varphi_{Z}\right) \\
& +2\left(2 N_{z} H_{y}+H_{y}\right) \cos \varphi_{Z} \sin \varphi_{Z}
\end{aligned}
$$

as in case (b), both terms disappear due to the gradient over the sample.

(e) The ${ }^{15} \mathrm{~N} x$-phase TROSY term in the antiecho sequence:

$$
\begin{aligned}
& 2 N_{x} H_{z}+N_{x} \stackrel{\text { gradient, pulses, delays, gradient }}{\longrightarrow} \\
& \left(H_{y}-2 N_{z} H_{y}\right) \cos \gamma_{N} B_{z, 1} \tau_{1} \cos \gamma_{H} B_{z, 2} \tau_{2} \\
& +\left(-H_{x}+2 N_{z} H_{x}\right) \cos \gamma_{N} B_{z, 1} \tau_{1} \sin \gamma_{H} B_{z, 2} \tau_{2} \\
& +\left(2 N_{z} H_{x}-H_{x}\right) \sin \gamma_{N} B_{z, 1} \tau_{1} \cos \gamma_{H} B_{z, 2} \tau_{2} \\
& +\left(2 N_{z} H_{y}-H_{y}\right) \sin \gamma_{N} B_{z, 1} \tau_{1} \sin \gamma_{H} B_{z, 2} \tau_{2} \\
& \text { if } \gamma_{N} B_{z, 1} \tau_{1}=\varphi_{Z} \quad \text { and } \quad \gamma_{H} B_{z, 2} \tau_{2}=-\varphi_{Z} \\
& \left(H_{y}-2 N_{z} H_{y}\right)\left(\cos \varphi_{Z} \cos \varphi_{Z}+\sin \varphi_{Z} \sin \varphi_{Z}\right) \\
& =\left(H_{y}-2 N_{z} H_{y}\right)
\end{aligned}
$$

As in the echo-sequence, we obtain that the slow relaxing ${ }^{15} \mathrm{~N}\left(2 N_{x} H_{z}+N_{x}\right)$ TROSY term transfers into the slow-relaxing ${ }^{1} \mathrm{H}\left(H_{y}-2 H_{y} N_{z}\right)$ (TROSY) term, but, importantly, of opposite sign.

(f) The ${ }^{15} \mathrm{~N}$ x-phase anti-TROSY term in the anti-echo sequence:

$$
\begin{aligned}
& 2 N_{x} H_{z}-N_{x} \stackrel{\text { Gradient, pulses, delays, gradient }}{\longrightarrow} \\
& \left(+H_{y}+2 N_{z} H_{y}\right) \cos \gamma_{N} B_{z, 1} \tau_{1} \cos \gamma_{H} B_{z, 2} \tau_{2} \\
& +\left(-H_{x}-2 N_{z} H_{x}\right) \cos \gamma_{N} B_{z, 1} \tau_{1} \sin \gamma_{H} B_{z, 2} \tau_{2} \\
& +\left(2 N_{z} H_{x}+H_{x}\right) \sin \gamma_{N} B_{z, 1} \tau_{1} \cos \gamma_{H} B_{z, 2} \tau_{2} \\
& +\left(2 N_{z} H_{y}+H_{y}\right) \sin \gamma_{N} B_{z, 1} \tau_{1} \sin \gamma_{H} B_{z, 2} \tau_{2} \\
& \text { if } \gamma_{N} B_{z, 1} \tau_{1}=\varphi_{Z} \quad \text { and } \quad \gamma_{H} B_{z, 2} \tau_{2}=-\varphi_{Z} \\
& \left(2 N_{z} H_{y}+H_{y}\right)\left(\sin \varphi_{Z} \sin \varphi_{Z}-\cos \varphi_{Z} \cos \varphi_{Z}\right) \\
& +2\left(H_{x}+2 N_{z} H_{x}\right) \cos \varphi_{Z} \sin \varphi_{Z}
\end{aligned}
$$

which, as shown above, both vanish over the sample.

(g) The ${ }^{15} \mathrm{~N}$ y-phase TROSY term in the antiecho-sequence: 
Table 3 Summary of transfers

\begin{tabular}{ccccc}
\hline $\begin{array}{c}\text { Coherence } \\
\text { in } t_{1}\end{array}$ & $\begin{array}{c}\text { ECHO } \\
\text { coherence in } t_{2}\end{array}$ & $\begin{array}{c}\text { Anti-Echo } \\
\text { Coherence in } t_{2}\end{array}$ & $\begin{array}{c}\text { Echo }+ \text { Anti-echo } \\
\text { Coherence in } t_{2}\end{array}$ & $\begin{array}{c}\text { Echo }- \text { Anti-Echo } \\
\text { Coherence in } t_{2}\end{array}$ \\
\hline $2 N_{\mathrm{x}} H_{\mathrm{z}}+N_{\mathrm{x}}$ & $-\left(H_{\mathrm{y}}-2 H_{\mathrm{y}} N_{\mathrm{z}}\right)$ & $+\left(H_{\mathrm{y}}-2 H_{\mathrm{y}} N_{\mathrm{z}}\right)$ & 0 & $-2\left(H_{\mathrm{y}}-2 H_{\mathrm{y}} N_{\mathrm{z}}\right)$ \\
$2 N_{\mathrm{x}} H_{\mathrm{z}}-N_{\mathrm{x}}$ & None & None & 0 & 0 \\
$2 N_{\mathrm{y}} H_{\mathrm{z}}+N_{\mathrm{y}}$ & $-\left(H_{\mathrm{x}}-2 H_{\mathrm{x}} N_{\mathrm{z}}\right)$ & $-\left(H_{\mathrm{x}}-2 H_{\mathrm{x}} N_{\mathrm{z}}\right)$ & $-2\left(H_{\mathrm{x}}-2 H_{\mathrm{x}} N_{\mathrm{z}}\right)$ & 0 \\
$2 N_{\mathrm{y}} H_{\mathrm{z}}-N_{\mathrm{y}}$ & None & None & 0 & 0 \\
\hline
\end{tabular}

$$
\begin{aligned}
& 2 N_{y} H_{z}+N_{y} \stackrel{\text { Gradient, pulses, delays, gradient }}{\longrightarrow} \\
& \left(+2 N_{z} H_{x}-H_{x}\right) \cos \gamma_{N} B_{z, 1} \tau_{1} \cos \gamma_{H} B_{z, 2} \tau_{2} \\
& +\left(2 N_{z} H_{y}-H_{y}\right) \cos \gamma_{N} B_{z, 1} \tau_{1} \sin \gamma_{H} B_{z, 2} \tau_{2} \\
& -\left(+H_{y}-2 N_{z} H_{y}\right) \sin \gamma_{N} B_{z, 1} \tau_{1} \cos \gamma_{H} B_{z, 2} \tau_{2} \\
& -\left(-H_{x}+2 N_{z} H_{x}\right) \sin \gamma_{N} B_{z, 1} \tau_{1} \sin \gamma_{H} B_{z, 2} \tau_{2} \\
& \text { if } \quad \gamma_{N} B_{z, 1} \tau_{1}=\varphi_{Z} \quad \text { and } \quad \gamma_{H} B_{z, 2} \tau_{2}=-\varphi_{Z} \\
& 2 N_{z} H_{x}-H_{x}
\end{aligned}
$$

As in the echo-sequence, the slow-relaxing ${ }^{15} \mathrm{~N}$ $\left(2 N_{y} H_{z}+N_{y}\right)$ TROSY term transfers into the slow-relaxing ${ }^{1} \mathrm{H}\left(H_{x}-2 H_{x} N_{z}\right)$ (TROSY) term, importantly, of the same sign.

(h) The ${ }^{15} \mathrm{~N}$ y-phase anti-TROSY term in the anti-echo sequence:

$$
\begin{aligned}
& 2 N_{y} H_{z}-N_{y} \stackrel{\text { Gradient, pulses, delays, gradient }}{\longrightarrow} \\
& \left(2 N_{z} H_{x}+H_{x}\right) \cos \gamma_{N} B_{z, 1} \tau_{1} \cos \gamma_{H} B_{z, 2} \tau_{2} \\
& +\left(2 N_{z} H_{y}+H_{y}\right) \cos \gamma_{N} B_{z, 1} \tau_{1} \sin \gamma_{H} B_{z, 2} \tau_{2} \\
& -\left(+H_{y}+2 N_{z} H_{y}\right) \sin \gamma_{N} B_{z, 1} \tau_{1} \cos \gamma_{H} B_{z, 2} \tau_{2} \\
& -\left(-H_{x}-2 N_{z} H_{x}\right) \sin \gamma_{N} B_{z, 1} \tau_{1} \sin \gamma_{H} B_{z, 2} \tau_{2} \\
& \text { if } \gamma_{N} B_{z, 1} \tau_{1}=\varphi_{Z} \text { and } \gamma_{H} B_{z, 2} \tau_{2}=-\varphi_{Z} \\
& \left(2 N_{z} H_{x}+H_{x}\right)\left(\cos \varphi_{Z} \cos \varphi_{Z}-\sin \varphi_{Z} \sin \varphi_{Z}\right) \\
& -2\left(2 N_{z} H_{y}+H_{y}\right) \cos \varphi_{Z} \sin \varphi_{Z}
\end{aligned}
$$

which, as shown above, both vanish over the sample.

We summarize the results for all coherences in Table 3 .

The table shows a complete ["sensitivity enhanced" $(7,8)]$ and pure transfer from the ${ }^{15} \mathrm{~N}$ TROSY transition to the ${ }^{1} \mathrm{H}$ TROSY transition in both the echo and anti-echo sequences. "Pure" here means that there is no cross talk between any of the terms. In practice, however, relaxation effects cause the different pathways to have different efficiencies, and small cross-terms can also arise due to crosscorrelated cross relaxation during the J-transfer modules of the sequence. These effects can be minimized (but not eliminated) by slight adjustments of the J-transfer timings (17).

The echo and anti-echo data is added and subtracted as is shown in Table 3 , and after a $90^{\circ}$ phase shift in the ${ }^{1} \mathrm{H}$ detection (i.e., exchange of $\mathrm{R}$ and $\mathrm{I}$ ) for the echo + anti-echo sum spectrum, a classic 2D hyper-complex FT can be performed (7).

In Fig. 6 we show the sensitivity-enhanced TROSY sequence as used in our laboratory. It has

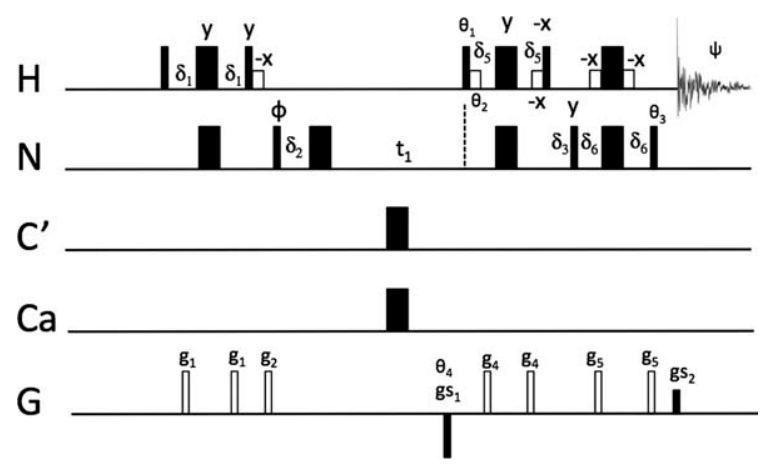

Figure $6 \quad 1 \mathrm{H}_{-}{ }^{15} \mathrm{~N}$ TROSY optimized for solvent suppression as used in the lab on Varian/Agilent instruments [extended from refs. (20) and (17)]. All phases are along $x$, unless indicated otherwise. Open boxes are sinebell pulses of $\sim 1.5 \mathrm{~ms}$ with a power level $\sim 40 \mathrm{~dB}$ below the hard-pulse power level, which serve to keep the $\mathrm{H}_{2} \mathrm{O}$ signal in $+z$ for most of the time. $\varphi=y,-y ; \psi=x,-x$. The phases for the echo/anti-echo pair are: $\theta_{1}=y,-y ; \theta_{2}$ $=-y, y ; \theta_{3}=x,-x ; \theta_{4}=-,+$. To shift residual axial peaks in $t_{1}$, phases $\varphi$ and $\psi$ are incremented by 180 degrees in the anti-echo transients [i.e., echo/anti-echo TPPI in analogy to states-TPPI (21)]. Because of this, the sequence can in principle be collected with just one transient per FID. In practice, carrying out the $\varphi=y,-y ; \psi$ $=x,-x$ phase cycle by allowing two transients per FID results in much cleaner spectra. $\delta_{1}, \delta_{5}$, and $\delta_{6}$ are $1 /$ $4 \mathrm{JNH}$, but can be adjusted for maximum sensitivity and cross-talk suppression (17). $\delta_{1}$ balances the $t_{1}$ timing; $\delta_{3}$ is equal to the duration of $\mathrm{gs}_{1}$ and $\mathrm{gs}_{2}(500 \mu \mathrm{s}) \cdot \mathrm{gs}_{1}=$ $16,000, \mathrm{gs}_{2}=1600$ (full power is $32 \mathrm{~K}$ ). The other gradients are set to $\sim 10 \%$ of full gradient power and are $1 \mathrm{~ms}$ in length. For Bruker instruments, the phase of the third proton pulse is set to $-\mathrm{y}$ and the sign of $\mathrm{gs}_{1}$ is inverted. 
been optimized for water suppression and phasing behavior and allows for decoupling of $J_{\mathrm{NCa}}$ and $J^{\prime}{ }_{\mathrm{NC}}$ in double/triple labeled proteins. Codes for Varian and for Bruker, with parameters, are available upon request.

\section{REFERENCES}

1. Pervushin K, Riek R, Wider G, Wüthrich K. 1997. Attenuated T2 relaxation by mutual cancellation of dipole-dipole coupling and chemical shift anisotropy indicates an avenue to NMR structures of very large biological macromolecules in solution. Proc Natl Acad Sci USA 94:12366-12371.

2. Pervushin K, Riek R, Wider G, Wüthrich K. 1998. Transverse Relaxation-Optimized Spectroscopy (TROSY) for NMR studies of aromatic spin systems in ${ }^{13} \mathrm{C}$ labeled proteins. J Am Chem Soc 120:6394-6400.

3. Sanders N.C.R., II, Schwonek JP. 1992. Characterization of magnetically orientable bilayers in mixtures of dihexanoylphosphatidylcholine and dimyristoylphosphatidylcholine by solid-state NMR. Biochemistry 31:8898-8905.

4. Salzmann M, Pervushin K, Wider G, Senn H, Wüthrich K. 1998. TROSY in triple-resonance experiments: new perspectives for sequential NMR assignment of large proteins. Proc Natl Acad Sci USA 95:13585-13590.

5. VandeVen FJM, Hilbers CW. 1983. A simple formalism for the description of multiple-pulse experimentsapplication to a weakly coupled 2 -spin $(I=1 / 2)$ system. J Magn Reson 54:512-520.

6. Sørensen OW, Eich GW, Levitt MH, Bodenhausen G, Ernst RR. 1983. Product operator-formalism for the description of NMR pulse experiments. Prog Nucl Magn Reson Spectrosc 16:163-192.

7. Palmer AG, III, Cavanagh J, Wright PE, Rance M. 1991. Sensitivity improvement in proton-detected 2-dimensional heteronuclear correlation NMR-spectroscopy. J Magn Reson 93:151-170.

8. Kay LE, Keifer P, Saarinen T. 1992. Pure absorption gradient enhanced heteronuclear single quantum correlation spectroscopy with improved sensitivity. J Am Chem Soc 114:10663-10665.

9. Goldman M. 1984. Interference effects in the relaxation of a pair of unlike spin 1/2 nuclei. J Magn Reson 60:437-452.

10. Werbelow LG. 1996. Relaxation Processes: Cross Correlation and Interference Terms in Encyclopedia of NMR. New York: John Wiley. p 4072-4078.

11. Fischer MWF, Majumdar A, Zuiderweg ERP. 1998. Protein NMR relaxation: theory, applications and outlook. Prog NMR Spectrosc 33:207-272.

12. Otting G, Soler LP, Messerle BA. 1999. Measurement of magnitude and sign of heteronuclear coupling constants in transition metal complexes. J Magn Reson 139:186-186.
13. Loth K, Pelupessy P, Bodenhausen G. 2005 Chemical shift anisotropy tensors of carbonyl, nitrogen, and amide proton nuclei in proteins through cross-correlated relaxation in NMR spectroscopy. J Am Chem Soc 127:6062-6068.

14. Werbelow LG, Grant DM. 1977. Determination of motional asymmetry of methyl rotators from ${ }^{13} \mathrm{C}$ spin dynamics. Adv Magn Reson 9:189-301.

15. Czisch M, Boelens R. 1998. Sensitivity enhancement in the TROSY experiment. J Magn Reson 134:158-160.

16. Loria JP, Rance M, Palmer AG, III. 1999. Transverse-relaxation-optimized (TROSY) gradientenhanced triple-resonance NMR spectroscopy. J Magn Reson 141: 180-184.

17. Schulte-Herbruggen T, Sørensen OW. 2000. Clean TROSY: compensation for relaxation-induced artifacts. J Magn Reson 144:123-128.

18. Yang DW, Kay LE. 1999. Improved (HN)-H-1detected triple resonance TROSY-based experiments. J Biomol NMR 13:3-10.

19. Otting G, Soler LP, Messerle BA. 1999. Measurement of magnitude and sign of heteronuclear coupling constants in transition metal complexes. J Magn Reson 137:413-429.

20. Rance M, Loria JP, Palmer AGR. 1999. Sensitivity improvement of transverse relaxation-optimized spectroscopy. J Magn Reson 136:92-101.

21. Marion D, Ikura M, Tschudin R, Bax A. 1989. Rapid recording of 2D NMR-spectra without phase cyclingApplication to the study of hydrogen-exchange in proteins. J Magn Reson 85:393-399.

\section{BIOGRAPHIES}

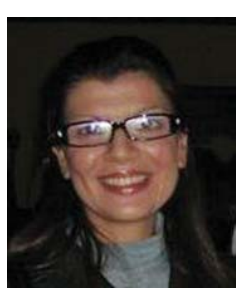

Dr. Aikaterini Rousaki is a graduate from the Biophysics Department of the University of Michigan with expertise in multidimensional NMR spectroscopy of proteins and their interactions with various drug targets. She received her PhD in April 2011, with Dr. Zuiderweg as advisor. She also holds a Masters in Applied Molecular Spectroscopy and more specifically light scattering from the University of Crete, Greece. She is currently a postdoctoral fellow at the BMRZ of the Goethe University, Frankfurt, and she is working on metastable states of the Sensory Rhodopsin II photo cycle using time-resolved NMR spectroscopy.

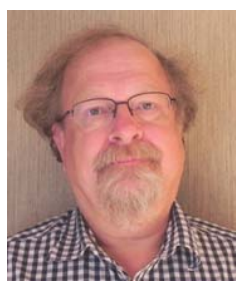

Professor Erik R.P. Zuiderweg obtained his $\mathrm{PhD}$ in biophysical chemistry from the University of Nijmegen, The Netherlands, and completed postdoctoral studies in protein NMR at the University of Groningen and the ETH-Zurich. Between 1985 and 1991 he was employed as a NMR research scientist at Abbott Laboratories, Chicago. Since 1991 he is Professor of Biological Chemistry at the University of Michigan. His current interests are in NMR experimental design with application to challenging systems such as the structure, dynamics, and interactions of the Hsp70 protein-folding chaperones. 